\title{
Development and evaluation of healthy cities
}

\author{
Kai Xia*, Yuheng Yao, Zhizhen Bu, and Zhijun Zhou \\ Alibaba Business School, Hangzhou Normal University, China
}

\begin{abstract}
The Healthy Cities and Sustainable Development Goals are interlinked, and the Healthy Cities movement has received widespread response and support worldwide over the past 30 years. The European Healthy Cities Networks covers about 1,400 cities. China started the Healthy Cities Movement in 1994 and put forward the Healthy China Strategy in the report of 19th national congress of CPC. Based on the impact of COVID-19 and Henan floods on urban health, it is necessary to incorporate some unconventional indicators into the Healthy Cities evaluation indicator framework. In addition, structured data cannot fully describe certain indicators, so the collection of unstructured data is equally important.
\end{abstract}

\section{Introduction}

\subsection{Healthy Cities and its definition}

Healthy Cities is a concept advocated by the World Health Organization (WHO) in 1984. It is a global movement that originated in Europe and has been established in all six WHO regions (https://www.euro.who.int/_data/assets/pdf_file/0011/285995/Healthy-Citiespromoting-health-and-equity.pdf). Since the 1980s, different institutions and scholars have tried to define Healthy Cities. In 1994, the WHO proposed a specific definition: A healthy city is one that continually creates and improves its physical and social environments and expands the community resources that enable people to mutually support each other in performing all the functions of life and developing to their maximum potential (https://www.euro.who.int/en/health-topics/environment-and-health/urban-health/whoeuropean) . Healthy Cities should not be limited to medical and health care, nor should it be shackled to urban construction, but should have the overall development of human beings as its core goal and value orientation [1]. Healthy Cities is a comprehensive concept that involves many aspects of urban construction and development, and requires the collaboration of departments and extensive social participation [2]. Healthy Cities is not confined to one or more health problems, but "is intended to build health into the decision-making processes of local governments, community organizations and business, to develop a broad range of strategies to address the broad social, environmental and economic determinants of health" [3]. In addition, there are many scholars who try to give concepts of healthy cities from different disciplinary perspectives.

\footnotetext{
*Corresponding author: xiakai96@163.com
} 


\subsection{Healthy cities projects and national healthy cities networks}

The first International Conference on Health Promotion was held in Ottawa in 1986. It launched a series of actions among international organizations, national governments and local communities to achieve the goal of "Health For All (HFA)" by the year 2000 and beyond [4]. As one of WHO's main vehicles for carrying out the strategy of HFA [5], WHO launched the Healthy Cities Projects (HCPs) in 1986, which gradually becoming a strategic tool for city management [6], and subsequently established the National Healthy Cities Networks (NNs) in the WHO European region. For 30 years, the NNs brought together about 100 flagship cities and 30 national networks, covering approximately 1,400 municipalities (https://www.euro.who.int/en/health-topics/environment-and-health/urban-health/whoeuropean-healthy-cities-network). As shown in Table 1, the development of the NNs consists of seven phases. Although divided into seven stages, they are actually coherent. It is a continuous enrichment and refinement of the initially established architecture.

Table 1. The phases of national healthy cities networks in the who European region.

\begin{tabular}{ll}
\hline Phase & Brief content \\
1988-1992(I) & setting up structures \\
1993-1997(II) & advanced the healthy cities approach \\
1998-2002(III) & transition from health promotion to integrated city health development plans \\
$2003-2008(\mathrm{IV})$ & overall commitment to health development \\
2009-2013(V) & priority was given to health and health equity in all policies \\
$2014-2018$ (VI) & $\begin{array}{l}\text { gives priority to life course approaches in city policies and plans } \\
\text { prioritize the themes presented in the Copenhagen Consensus of Mayors }\end{array}$ \\
\hline
\end{tabular}

\subsection{Healthy cities and 2030 agenda}

In September 2015, the United Nations Sustainable Development Summit adopted one of the UN Summit Outcomes, "Transforming our World: 2030 Agenda for Sustainable Development". The document proposes 17 Sustainable Development Goals (SDGs) and 169 targets. The goals and targets encompass the three dimensions of sustainable development: economic, social and environmental, which are in line with the main theme of Healthy Cities. Most of the goals are related to Healthy Cities, especially goals 2, 3, 6, 7 and 11, in other words Healthy Cities are an important means or vehicle to achieve these goals.

Thus, the construction of Healthy Cities has become increasingly important and is an inevitable requirement for sustainable human development.

\section{Healthy cities in China}

In 1994, China introduced the concept of Healthy Cities, which is different from Sanitary Cities and brings us a multidimensional and comprehensive perspective. In fact, the Healthy Cities Movement is an upgrade of the Patriotic Health Movement. In China, the Healthy Cities Projects was first piloted in Dongcheng District, Beijing and Jiading District, Shanghai. The construction of Healthy Cities in China has gone through two stages of exploration and substantial development, and the turning point was SARS in 2003 [7]. In November 2016, the Patriotic Health Office identified the first 38 pilot cities for the Healthy Cities Projects, and since then the Healthy Cities Movement has been fully implemented in China. 
In October 2017, the 19th National Congress of the CPC adopted the major decision for carrying out the Health China Strategy. Healthy Cities are an effective path to achieve the Healthy China Strategy, and the Healthy China Strategy is also an expansion of the Healthy Cities Projects, which also reflects the importance the Chinese government attaches to national health. In July 2019, the General Office of the State Council of China established the "Health China Action Promotion Committee", which is responsible for coordinating and promoting the work related to the organization and implementation, testing and assessment of the "Health China Action (2019-2030)".

According to the China Urban Development Report 2019, only 35 of the 288 cities above the prefecture level are in a relatively healthy state of development, healthy cities account for only $12 \%$, and more than $80 \%$ of cities are in a "sub-healthy" state. It is clear that the health of Chinese cities still faces serious challenges in general, so it is necessary to promote Healthy Cities Projects in China.

\section{Healthy cities evaluation}

Good evaluation can ensure that the assessment of healthy city construction is more oriented, scientific and feasible, thus promoting the sustainable construction of Healthy Cities [8]. When it comes to Healthy Cities assessment, two points need to be noted, one is to establish appropriate indicators and the other is to assign weights to different indicators reasonably.

\subsection{Establish an appropriate indicator system}

The NNs developed a set of 53 Healthy Cities Indicators (HCIs), based on which the HCPs improved the original indicators and reduced them to 32 , while improved the original definitions. The collection and analysis of indicators of HCIs were to: provide a description of health in the city and provide a baseline of information to make comparisons over time [9]. Well-designed and carefully selected indicators can help a city determine its level of development, as well as clarify how close it is to its goals.

The China Healthy Cities Evaluation Index System, released in 2018, contains 42 specific indicators. In fact, many Chinese cities, such as Hangzhou, Qingdao and Shenzhen, have also proposed different Healthy City index. However, the proposed indicators are all structured data. Although the HCIs proposed by NNs contain a small amount of qualitative data, they are also semi-structured data.

The selection of indicators requires attention to the following points:

(1) A simple indicator system may not provide a comprehensive view of healthy cities, while a complex indicator system will bring difficulties to data collection, so it is especially important to establish a scientific and practical indicator system that can be put into practice.

(2) The definitions of some indicators differ between cities, which may affect the comparison between different cities.

Compared with unstructured data, structured data are easier to collect and process, but structured data may ignore certain critical factors. Therefore, we will try to obtain unstructured data related to Healthy Cities from social platforms such as Weibo to supplement the structured data and thus describe the indicators more accurately.

\subsection{Assign weights to different indicators}

A set of indicators generally contains many aspects, and evaluating the health of a city is never a simple addition or subtraction of the indicators. Different indicators contribute to the 
health of a city in different ways, so how to assign weights to different indicators is another important task after determining the indicator system.

There are various different methods to assign weights, subjective and objective method in the evaluation of Healthy Cities, such as comprehensive index method, rank-sum ratio method (RSR), entropy method, integrated RSR, etc. Comprehensive index method can be recommended as an appropriate method applying in healthy cities evaluation based on normative Delphi studies [10]. The rationality of the Entropy Weight Method (EWM) in decision-making is questionable [11]. Different weighting methods may lead to different evaluation results. Therefore, comparing the feasibility and effectiveness of various methods and using appropriate weighting methods is one of the main tasks next.

\subsection{Add unconventional indicators to indicator framework}

The COVID-19 epidemic outbreak in 2019 and the floods in Henan Province in 2021 show us how vulnerable our cities are under unexpected event states. Emergencies may only happen once every few years or even a dozen years, but they have a profound and long-lasting impact on the normal functioning of cities. Therefore, it is necessary to consider adding forecasting and processing indicators to the indicator framework. Combined with the contents in 3.1, we propose a Healthy Cities Indicator Framework including unconventional situations as shown in Figure. 1. It is worth noting that emergency management is not only physical treatment, but the psychological state of the population also needs attention. As we saw during the COVID-19 epidemic outbreak, public opinion may cause secondary disasters thereby amplifying and prolonging the negative impact of the outbreak.

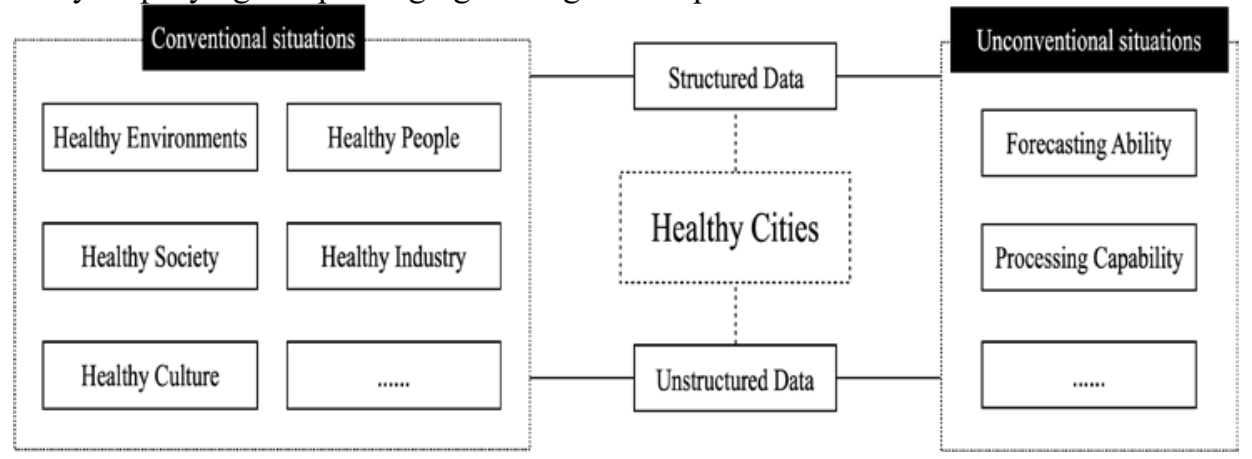

Fig. 1. Healthy Cities Indicator Framework.

Although the HCPs has been in existence for more than 30 years, very little research has been done on Healthy Cities under unconventional situations. We believe that after the COVID-19 epidemic, more and more scholars, institutions, and governments will pay attention to the research of incorporating non-conventional indicators into the Healthy Cities indicator framework.

\section{Conclusion}

Healthy Cities is not only a goal, but also a process that requires continuous efforts and improvements. Healthy Cities is not the business of a certain department or organization. We hope that the promotion of the HCPs will strengthen the cooperation among various government departments and provide a broader perspective for the construction of Healthy Cities. 
A review of Healthy Cities and HCPs shows that they are consistent with the SDGs. The current description of Healthy Cities is almost structured indicator data, and it is obvious that the structured data may lead us to miss some important information. In order to create more robust Healthy Cities, the Healthy Cities Indicator Framework should take into account both conventional and non-conventional situations.

\section{References}

1. G.W. Huang, Journal of Northwest University (Philosophy and Social Sciences Edition), 48,74-82 (2018)

2. H.N. Yu, G. Cheng, H.P. Wang, J. Chang, Q.Y. Meng, Chinese JHP,5,30-33 (2012)

3. B.C. Flynn, Annu Rev. Public Health, 17, 299-309 (1996)

4. Ottawa Charter for Health Promotion (1986)

5. A.D. Tsouros, HEAKTH PROMOTION INTERNATIONAL, 10, 2 (1996)

6. X.H. Zhou, Journal of Public Management, 3,68-73 (2006)

7. H.F, Medicine \& Philosophy, 27, 12-15, (2006)

8. W.J. Huang, R.X. Bai, P. Hu, Z. Lin, etc., Medicine \& Philosophy, 38, 56-59, (2017)

9. P.Webster, D. Sanderson, Journal of Urban Health, 90, S52-S61(2012)

10. L.Ran, X.D. Tian, Y. Xu, K.Y. Zhang, etc., SCS,65,102581, (2021)

11. Y.X. Zhu, D.Z. Tian, F. Yan, Math. Probl. Eng., 2020, 3564835(2020) 